\title{
Extremely Low-Frequency Electromagnetic Fields Affect Myogenic Processes in C2C12 Myoblasts: Role of Gap-Junction-Mediated Intercellular Communication
}

\author{
Caterina Morabito, ${ }^{1}$ Nathalie Steimberg, ${ }^{2}$ Francesca Rovetta, ${ }^{2}$ Jennifer Boniotti, ${ }^{2}$ \\ Simone Guarnieri, ${ }^{1}$ Giovanna Mazzoleni, ${ }^{2}$ and Maria A. Mariggiò ${ }^{1}$ \\ ${ }^{1}$ Department of Neuroscience, Imaging and Clinical Sciences, Unit of Functional Biotechnology and StemTeCh Group, \\ Centro Scienze dell' Invecchiamento e Medicina Traslazionale (CeSI-MeT), "G. d'Annunzio" University of Chieti-Pescara, Chieti, Italy \\ ${ }^{2}$ Tissue Engineering Unit, Anatomy and Physiopathology Division, Department of Clinical and Experimental Sciences, \\ School of Medicine, University of Brescia, Brescia, Italy
}

Correspondence should be addressed to Caterina Morabito; cmorabit@unich.it

Received 10 March 2017; Accepted 19 April 2017; Published 21 May 2017

Academic Editor: Brad Upham

Copyright (c) 2017 Caterina Morabito et al. This is an open access article distributed under the Creative Commons Attribution License, which permits unrestricted use, distribution, and reproduction in any medium, provided the original work is properly cited.

\begin{abstract}
Extremely low-frequency electromagnetic fields (ELF-EMFs) can interact with biological systems. Although they are successfully used as therapeutic agents in physiatrics and rehabilitative practice, they might represent environmental pollutants and pose a risk to human health. Due to the lack of evidence of their mechanism of action, the effects of ELF-EMFs on differentiation processes in skeletal muscle were investigated. C2C12 myoblasts were exposed to ELF-EMFs generated by a solenoid. The effects of ELF-EMFs on cell viability and on growth and differentiation rates were studied using colorimetric and vital dye assays, cytomorphology, and molecular analysis of MyoD and myogenin expression, respectively. The establishment of functional gap junctions was investigated analyzing connexin 43 expression levels and measuring cell permeability, using microinjection/dye-transfer assays. The ELF-EMFs did not affect $\mathrm{C} 2 \mathrm{C} 12$ myoblast viability or proliferation rate. Conversely, at ELF-EMF intensity in the mT range, the myogenic process was accelerated, through increased expression of MyoD, myogenin, and connexin 43. The increase in gap-junction function suggests promoting cell fusion and myotube differentiation. These data provide the first evidence of the mechanism through which ELF-EMFs may provide therapeutic benefits and can resolve, at least in part, some conditions of muscle dysfunction.
\end{abstract}

\section{Introduction}

Modern industrial society produces considerable levels of electromagnetic fields (EMFs), caused by widespread use of electric-power-generating facilities that deliver energy to domestic and industrial appliances. These EMFs are characterized by frequencies of $50 \mathrm{~Hz}$ to $60 \mathrm{~Hz}$ and are part of the extremely low-frequency (ELF) electromagnetic spectrum [1].

Even if ELF-EMFs are nonionizing radiation, they can interact with biological matter [2]. Since the 1980s, chronic exposure to ELF-EMFs has been implicated in carcinogenesis $[3,4]$, even if epidemiological studies have not provided conclusive or statistically adequate data. This has mainly been due to confounding by variable exposure times and misclassification selection bias $[5,6]$.

On the other hand, ELF-EMF-based therapies are commonly used in different medical fields, such as physiatrics and patient rehabilitation [7-9], with apparent beneficial effects. However, at present, the cellular mechanism(s) behind these effects remains unclear. Consequently, experimental investigations addressing how, and to what extent, ELF-EMFs influence living matter become urgent.

Our studies have been specifically designed to provide new insight into the mechanism(s) responsible for the biological effects of ELF-EMFs on skeletal muscle. We previously described the effects of ELF-EMFs on the C2C12 myoblast cell line [10], a well-known in vitro model of skeletal muscle 
phenotype [11]. We demonstrated that ELF-EMFs affect intracellular reactive oxygen species production, mitochondrial membrane potential, and $\mathrm{Ca}^{2+}$ handling, which have been shown to be all patterns strictly dependent on the cell differentiation stage in muscle tissue [12]. Such ELF-EMF-induced changes in mitochondrial activity and $\mathrm{Ca}^{2+}$ homeostasis support the hypothesis that exposure to ELF-EMFs can effectively modulate regulation of the myogenesis in $\mathrm{C} 2 \mathrm{C} 12$ myoblasts.

Skeletal myogenesis is required for growth, maintenance, and repair of injured muscle [13]. It is a multistep developmental process determined by a complex cascade of events involving development and differentiation of myoblasts, their fusion to form primary and secondary myotubes, and their subsequent maturation into fully developed adult muscle fibers $[14,15]$.

In recent years, several studies have led to significant improvements in the definition and understanding of the phases of the commitment and differentiation processes of skeletal muscle cells [15-19]. Many in vitro studies have focused on the role of gap-junction-mediated intercellular communication (GJIC) in specific and critical stages of myogenesis [20-23]. It has been hypothesized that GJIC might be involved in the onset of the differentiation process, to coordinate the myoblasts that are committed to differentiate, thus promoting their alignment and fusion, and, consequently, the intercellular diffusion of critical signaling molecules (i.e., $\mathrm{Ca}^{2+}$, inositol 1,4,5-trisphosphate, and adenosine $5^{\prime}$ triphosphate) $[21,24,25]$. The evidence that short exposure to ELF-EMFs has effects on both $\mathrm{C} 2 \mathrm{C} 12$ myoblasts and myotubes through modulation of their redox status and $\mathrm{Ca}^{2+}$ handling [10] also suggests a possible involvement of GJIC and, as a consequence, of long-term biological processes, like myogenesis [26].

To further clarify the mechanism(s) responsible for ELFEMF-induced effects on myogenesis, in the present study we exposed differentiating C2C12 myoblasts to ELF-EMFs, and, in particular, we monitored their gap-junction permeability, which is considered to be a crucial function for myotube formation during progression through myogenesis.

\section{Materials and Methods}

2.1. Equipment. A horizontal solenoid (Oersted Technology Corporation) was designed and built to deliver variable, homogeneous, sine-wave alternating-current magnetic fields at $50 \mathrm{~Hz}$ frequency and with intensities from $0.1 \mathrm{mT}$ to $1.0 \mathrm{mT}$ $( \pm 2 \%)$. This was used to expose large numbers of cells to ELFEMFs simultaneously [10, 27, 28]. The horizontal cylindrical solenoid presents the following features: length, $340 \mathrm{~mm}$; diameter, $113 \mathrm{~mm}$; number of wire turns, 144; resistance at $20^{\circ} \mathrm{C}(\Omega), 0.3$; this device was mounted on a nonmagnetic supporting base and powered using a power supply (CW801P; Elgar Electronics). In particular, the power supply was operated in its "closed-loop current mode": the output current and the generated magnetic field were consequently regulated against thermal and physical variations/drift of the coil. The final measured value of the solenoid coil constant was 4.830 Gauss/Amp. The solenoid was used in a cell culture incubator $\left(5 \% \mathrm{CO}_{2}, 37^{\circ} \mathrm{C}\right.$; HERAcell, Kendro Laboratory Products $\mathrm{GmbH}$, Hanau, Germany) for long periods, providing continuous exposure of the cells (1-7 days). During these treatments, any temperature increase in the incubator due to the solenoid was negligible. The tested value of background electromagnetic fields was less than $1 \mu \mathrm{T}(50 \mathrm{~Hz})$. In particular it was in the order of $0.7 \mu \mathrm{T}(50 \mathrm{~Hz})$ in the incubator and outside the switched on solenoid and of $100 \mathrm{nT}(50 \mathrm{~Hz})$ outside or inside the switched off solenoid placed in the incubator. Moreover, in laboratory areas between incubators, worktops, and cell cultures hood, electromagnetic fields measured 40-140 nT (50 Hz). The EMF meters used to measure the values of electromagnetic fields are F.W.BELL Tesla meters mod. 4190 (measuring range: $0.01-200 \mu \mathrm{T}$, resolution: $0.01 \mu \mathrm{T}$ ) and mod. 6010 equipped with an axial probe mod. HAD61-250805T (measuring range: $0.3-300 \mathrm{mT}$, minimum resolution: $0.01 \mathrm{mT}$ ) both from Sypris Test \& Measurement (Orlando, FL). The cells were cultured in plastic dishes that are transparent to the ELF magnetic field.

2.2. Chemicals and Materials. Unless otherwise indicated, the cell culture media, sera, and antibiotics were from Thermofisher (Monza, Italy), the cell culture plasticware was from Becton Dickinson Falcon (Sacco Srl, Cadorago, Italy), and the reagents and standards were from Sigma-Aldrich (Milan, Italy).

2.3. Cell Culture. Undifferentiated $\mathrm{C} 2 \mathrm{C} 12$ cells (myoblasts; CRL 1772; American Type Culture Collection, Manassas, VA, USA) were maintained routinely in growth medium (Dulbecco's modified Eagle's medium, 20\% fetal bovine serum, $4 \mathrm{mML}$-glutamine, $100 \mathrm{IU} / \mathrm{mL}$ penicillin, and $100 \mu \mathrm{g} / \mathrm{mL}$ streptomycin). The differentiated phenotype of these $\mathrm{C} 2 \mathrm{C} 12$ myoblasts (i.e., myotubes) was obtained by culturing these cells in differentiation medium (Dulbecco's modified Eagle's medium, 2\% heat-inactivated horse serum, $4 \mathrm{mML}$-glutamine, $100 \mathrm{IU} / \mathrm{mL}$ penicillin, and $100 \mu \mathrm{g} / \mathrm{mL}$ streptomycin) for 7 days to 10 days. Both undifferentiated and differentiating $\mathrm{C} 2 \mathrm{C} 12$ myoblasts were routinely maintained at $37^{\circ} \mathrm{C}$ in a humidified $5 \% \mathrm{CO}_{2}$ atmosphere.

2.4. Viability and Proliferation Assays. The analysis of cell viability was performed using trypan blue exclusion tests and cell growth using colorimetric assays based on the reduction of 3-(4,5-dimethylthiazol-2-yl)-2,5-diphenyltetrazolium bromide (MTT). Briefly, C2C12 myoblasts $\left(4.0 \times 10^{3}\right.$ cells $\left./ \mathrm{cm}^{2}\right)$ were plated into 96-well plates (Corning-Costar, Milan, Italy) in growth medium. At selected times with the absence and presence of the ELF-EMF treatments, MTT was added to each well to a final concentration of $0.5 \mathrm{mg} / \mathrm{mL}$. After $3 \mathrm{~h}$ at $37^{\circ} \mathrm{C}$, the plates were centrifuged at $500 \times \mathrm{g}$ for $5 \mathrm{~min}$. The supernatants were removed, and $200 \mu \mathrm{L}$ dimethyl sulfoxide was added to each well. After $30 \mathrm{~min}$ at $37^{\circ} \mathrm{C}$, the absorbance of each well was determined using a microplate reader (SpectraMAX 190), at $560 \mathrm{~nm}$. The trypan blue exclusion assay was performed by staining $\mathrm{C} 2 \mathrm{C} 12$ myoblasts with a trypan blue dye solution ( $0.5 \%$ in phosphate-buffered saline), and the 
stained cells were counted using a hemocytometer (Bürker chamber). Blue stained cells were considered nonviable.

2.5. Fusion Index. The extent of successful differentiation of C2C12 myoblasts into myotubes was determined by morphological analysis and Hoechst staining of the nuclei [29]. C2C12 myoblasts $\left(4.0 \times 10^{3}\right.$ cells $\left./ \mathrm{cm}^{2}\right)$ were seeded in growth medium onto sterile coverslips $(\varnothing, 12 \mathrm{~mm})$ in 24 -well plates. After 3 days, the growth medium was shifted to differentiation medium, and the $\mathrm{C} 2 \mathrm{C} 12$ myoblasts were induced to differentiate for up to 7 days in the absence and presence of the ELFEMF treatments. At each time point, the cells were washed twice with phosphate-buffered saline, fixed in 90\% ethanol, and stained with Hoechst solution. Images were acquired using an inverted microscope (Olympus IX-70) equipped with a digital camera (Camedia C-5050; Olympus). At least 20 fields were analyzed for each experimental condition. The Fusion Index was quantified as the percentage of Hoechststained nuclei located within multinucleated cells (with at least two nuclei, as a result of myoblast fusion), based on the total analyzed nuclei.

2.6. Western Blotting. C2C12 myoblasts $\left(4.0 \times 10^{3}\right.$ cells $\left./ \mathrm{cm}^{2}\right)$ were seeded in growth medium into $100 \mathrm{~mm}$ diameter Petri dishes. After 3 days, the growth medium was shifted to differentiation medium, and the cells were maintained in the absence and presence of the ELF-EMF treatments. At each experimental point, the cells were scraped, lysed, and collected in sample buffer $(62.5 \mathrm{mM}$ Tris- $\mathrm{HCl}, \mathrm{pH} 6.8,2 \%$ SDS, $10 \%$ glycerol, $0.1 \mathrm{M}$ dithiothreitol, and $0.002 \%$ bromophenol blue). Protein concentrations were determined using the protein assay kits (Bio-Rad DC; Bio-Rad, Segrate, Italy). The whole cell extracts were separated using SDS-PAGE on 7.5\% or $12.5 \%(\mathrm{w} / \mathrm{v})$ homogeneous slab gels ( $40 \mu \mathrm{g}$ protein/lane) and then transferred onto polyvinylidene fluoride membranes (Immobilon-P; Merck-Millipore, Vimodrone, Italy). Equal loading of protein samples was monitored using Coomassie blue staining of identical gels run in parallel $(0.25 \%$ Coomassie blue solution; R 250/G 250 1:1; Bio-Rad). Red Ponceau S (Sigma-Aldrich) staining of each membrane was used to monitor the transfer efficiency.

The membranes were hybridized with a mouse monoclonal anti-connexin 43 (cx43) antibody (dilution, 1:500; Chemicon International Inc., Temecula, CA, USA), a rabbit polyclonal anti-MyoD antibody (dilution, 1:500; Santa Cruz Biotechnology Inc., Santa Cruz, CA, USA), or a mouse monoclonal anti-myogenin antibody (dilution, 1:500; Santa Cruz Biotechnology Inc.), followed by reaction with horseradishperoxidase-conjugated anti-mouse or anti-rabbit IgGs (1:10,000; GE Healthcare, Cologno Monzese, Italy). The membranes were then incubated with horseradish-peroxidase conjugated anti-IgG, with the relevant proteins detected using chemiluminescence kits (Pierce EuroClone S.p.A., Pero, Italy) and an image acquisition system (Uvitec, Cambridge, UK). An anti-ERK1/2 antibody (1:2000 dilution; Santa Cruz Biotechnology Inc.) was used as the loading control.
2.7. Microinjection/Dye-Transfer Assay. The differentiating C2C12 myoblasts were tested for their establishment of functional gap junctions using a microinjection/dye-transfer assay, following the protocol proposed by Mazzoleni et al. [30]. Briefly, C2C12 myoblasts $\left(4.0 \times 10^{3}\right.$ cells $\left./ \mathrm{cm}^{2}\right)$ were seeded in growth medium into $60 \mathrm{~mm}$ diameter Petri dishes. After 3 days, the growth medium was shifted to differentiation medium, and the cell cultures were maintained in the absence and presence of the ELF-EMF treatments. For the dye-transfer assay, single cells within a monolayer were microinjected with a $10 \%(\mathrm{w} / \mathrm{v})$ solution of the gap-junction-permeant fluorescent tracer lucifer yellow $\mathrm{CH}$ in $0.33 \mathrm{M}$ lithium chloride. Microinjections were performed using glass capillary needles (Clark Electromedical Instruments, Edenbridge, UK) prepared in an automatic puller (Narishige, Tokyo, Japan). The needles were driven using a micromanipulator (Narishige 5240) linked to a fluorescence microscope (Olympus IMT2SYFII). The fluorescent dye was injected into single cells under nitrogen pressure, using a microinjector (Eppendorf, Hamburg, Germany). Five minutes after the last injection, the cells were fixed with $4 \%$ paraformaldehyde in phosphatebuffered saline, and their dye-transfer was evaluated. The extent of functional GJIC was quantified by counting the number of fluorescent cells surrounding the microinjected cells (i.e., the number of dye-coupled cells/injection). For the precise quantification of functional GJIC in the cultures, at least 25 independent microinjection trials/dish were carried out in three separate dishes for each experimental point. Functional GJIC is expressed as the means \pm SEM.

Fluorescence and phase-contrast images of untreated and ELF-EMF-treated monolayers were obtained using the inverted fluorescence microscope (Olympus IMT2-SYFII) equipped with a digital camera (Olympus OM-4 Ti reflex).

2.8. Statistical Analyses. Statistical analysis was performed using the Prism 4 software for Windows (GraphPad Software Inc., San Diego, CA, USA). Unless otherwise indicated, all of the data are expressed as means \pm SD or \pm SEM, as specified in the figure legends. Comparisons were made using $t$-tests.

\section{Results}

3.1. Effects of ELF-EMF Exposure on C2C12 Myoblast Proliferation and Differentiation. The effects of ELF-EMF exposure on the $\mathrm{C} 2 \mathrm{C} 12$ myoblast growth rate and viability were studied using a colorimetric assay and vital dye staining, respectively. These analyses were performed on undifferentiated $\mathrm{C} 2 \mathrm{C} 12$ myoblast cultures exposed for up to 7 days to ELF-EMF treatments at different field intensities $(0.1,0.5$, and $1.0 \mathrm{mT})$. As shown in Figure 1, exposure of the $\mathrm{C} 2 \mathrm{C} 12$ myoblasts to these ELF-EMFs did not significantly affect either cell proliferation rates or viability; indeed, the numbers of nonviable cells did not differ between the ELF-EMF-treated C2C12 myoblast cultures and their corresponding untreated controls and remained from $10 \%$ to $15 \%$ of the total cells.

The effect of ELF-EMF exposure on the myogenesis process of the $\mathrm{C} 2 \mathrm{C} 12$ myoblasts was explored using morphological analysis and quantification of the expression of MyoD and 


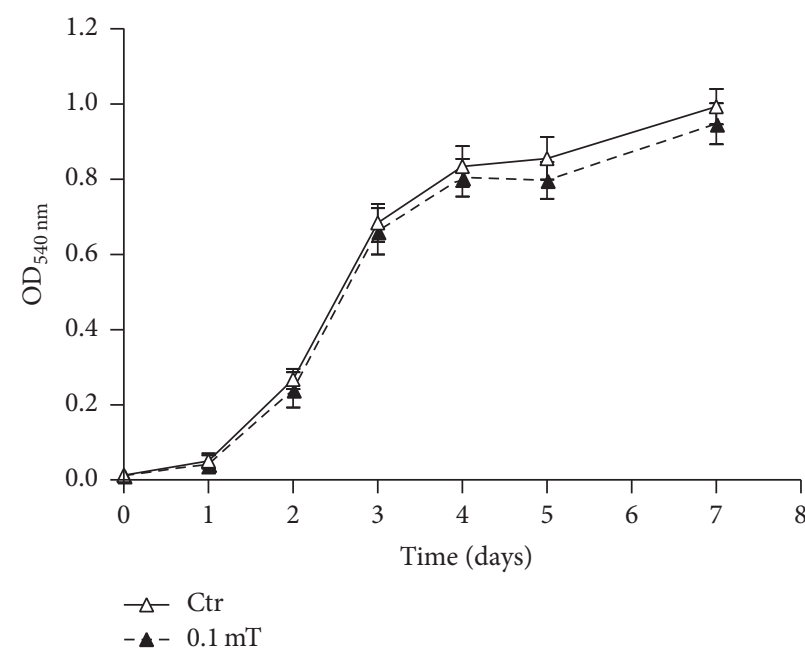

(a)

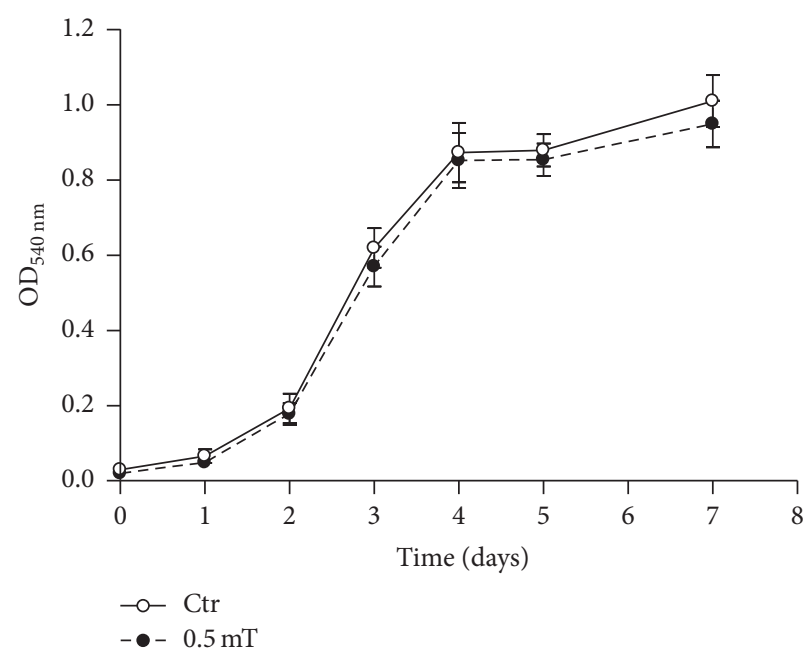

(b)

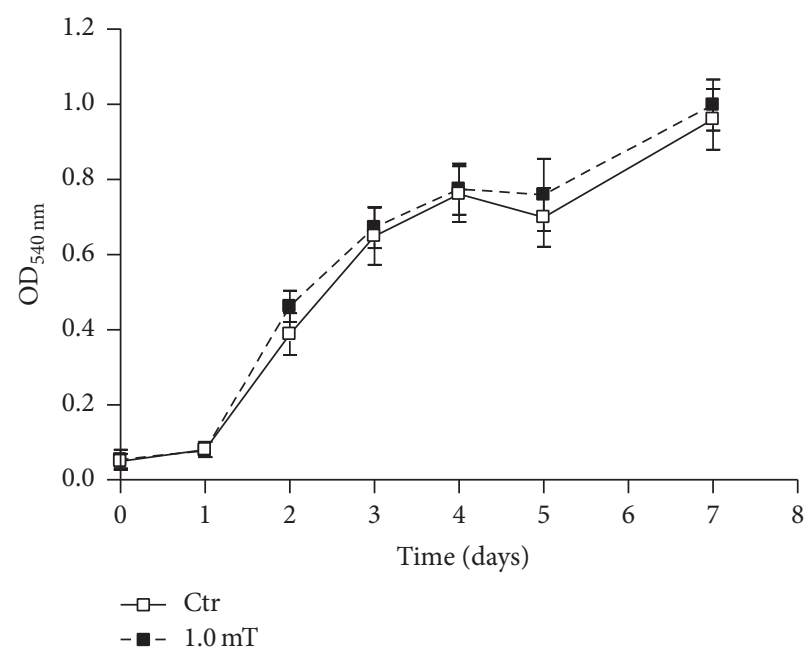

(c)

FIGURE 1: Effects of ELF-EMF treatments on C2C12 myoblast proliferation. Cell proliferation curves derived from MTT colorimetric assays performed on $\mathrm{C} 2 \mathrm{C} 12$ myoblasts in the absence (Ctr) and presence of ELF-EMF treatments with $0.1 \mathrm{mT}$ (a), $0.5 \mathrm{mT}$ (b), and $1.0 \mathrm{mT}$ (c). Data are means \pm SD from two independent experiments, each performed in six independent culture wells $(n=12)$.

myogenin, two early markers of myogenesis. The morphological analysis was quantified according to the Fusion Index, which was calculated as the percentage of $\mathrm{C} 2 \mathrm{C} 12$ myoblasts (of the total) that underwent the differentiation process. The exposure to ELF-EMFs at the $0.5 \mathrm{mT}$ and $1.0 \mathrm{mT}$ intensities induced significant acceleration of the first phases of the myogenic process. Indeed, the $\mathrm{C} 2 \mathrm{C} 12$ myoblasts showed significantly higher Fusion Index when exposed to ELF-EMF treatments with $0.5 \mathrm{mT}$ after 2 days to 5 days and with $1.0 \mathrm{mT}$ after 2 days to 3 days (Figures 2(b) and 2(c)). No significant differences were observed for the $\mathrm{C} 2 \mathrm{C} 12$ myoblast cultures exposed to ELF-EMFs at $0.1 \mathrm{mT}$, in comparison with the untreated controls (Figure 2(a)).

MyoD and myogenin expression levels showed some increases for the C2C12 myoblasts exposed to ELF-EMF treatments, with respect to the corresponding control cells (Figure 3 ). For the ELF-EMF treatment with $0.1 \mathrm{mT}$, this increase was evident only for myogenin expression after 72 hours of exposure. The exposure to $0.5 \mathrm{mT}$ intensity triggered an increase of MyoD expression at 48 hours and of myogenin expression at 24 and 48 hours. Differently, $1.0 \mathrm{mT}$ induced increased MyoD expression at 24 hours and increased myogenin expression at 72 hours of exposure.

\subsection{Influence of ELF-EMFs Treatments on C2C12 Myoblast} cx43 Expression and GJIC. The effects of ELF-EMF treatments on differentiating $\mathrm{C} 2 \mathrm{C} 12$ myoblasts were also evaluated by an analysis of the efficiency of their gap-junction coupling (dye-transfer efficiency) and by determining their expression levels of cx43, the major gap-junction component in skeletal myoblasts [20, 31, 32].

As illustrated by the representative Western blot and densitometric analyses in Figure 4, during the ELF-EMF treatment with $0.1 \mathrm{mT}$, a transient, but not significant, increase in 

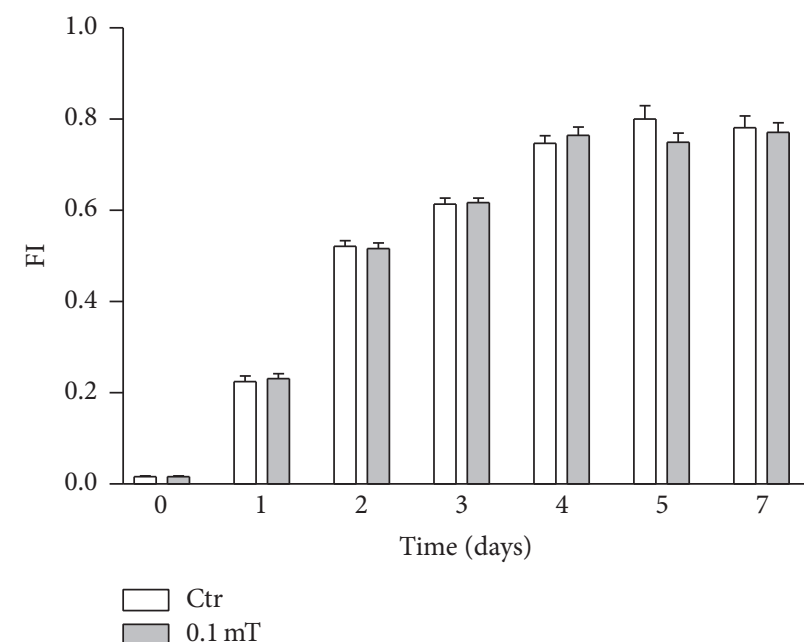



$\square$ Ctr $0.5 \mathrm{mT}$

(a)

(b)

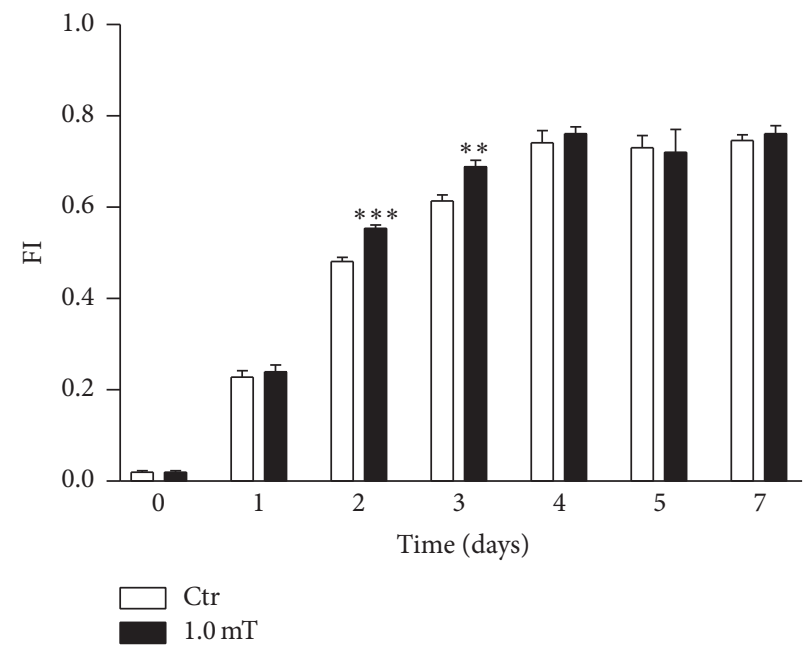

(c)

FIGURE 2: Effects of ELF-EMF treatments on the C2C12 myoblast myogenic process evaluated in the morphological analysis. Percentages of differentiating C2C12 myoblasts expressed as the Fusion Index (see Materials and Methods), as quantified in C2C12 myoblast cultures incubated in the absence (Ctr) and presence of ELF-EMF treatments with $0.1 \mathrm{mT}(\mathrm{a}), 0.5 \mathrm{mT}(\mathrm{b})$, and $1.0 \mathrm{mT}(\mathrm{c})$. Data are means \pm SEM from two independent experiments, each performed in six independent culture wells $(n=12) .{ }^{*} p<0.05,{ }^{* *} p<0.01$, and ${ }^{* * *} p<0.001$.

cx43 expression was seen only at 24 hours, with respect to the corresponding control cells. Conversely, when the cells were exposed to ELF-EMF treatment with $0.5 \mathrm{mT}$, or $1.0 \mathrm{mT}$, these showed an increase in cx43 expression that became significant at 72 hours, with respect to the corresponding control cells (Figure 4).

The influence of the ELF-EMF treatment on C2C12 myoblast gap-junction function was tested using the microinjection/dye-transfer assay at the early stages of $\mathrm{C} 2 \mathrm{C} 12$ myoblast differentiation (i.e., 24, 48 h). These times were selected on the basis that it is the earlier times that are crucial for the onset of the differentiation program in these $\mathrm{C} 2 \mathrm{C} 12$ myoblasts. In addition, after $48 \mathrm{~h}$, the high cell density that was reached by the $\mathrm{C} 2 \mathrm{C} 12$ myoblast monolayers made the single-cell approach for the assay difficult. The quantitative analysis of the extent of dye-coupling during the first $48 \mathrm{~h}$ of differentiation of the $\mathrm{C} 2 \mathrm{C} 12$ myoblasts (e.g., see the representative phase contrast and corresponding fluorescence images in panel (a) and the graph in panel (b) of Figure 5) revealed an increased number of coupled cells in the cultures exposed to ELF-EMF treatment that became significant after $48 \mathrm{~h}$ at $1.0 \mathrm{mT}$, in comparison to the corresponding controls.

The treatment of the differentiating $\mathrm{C} 2 \mathrm{C} 12$ myoblasts with ELF-EMFs at 0.5 or $1.0 \mathrm{mT}$ increased not only the expression of the major junction component, cx43, but also its assembly into functional membrane channels, which allowed direct cell-to-cell communication among the cells that were committed to differentiate.

\section{Discussion}

Epidemiological studies on the potential hazards of EMFs for human health have generated many controversies and attracted the attention of the media, the general public, 


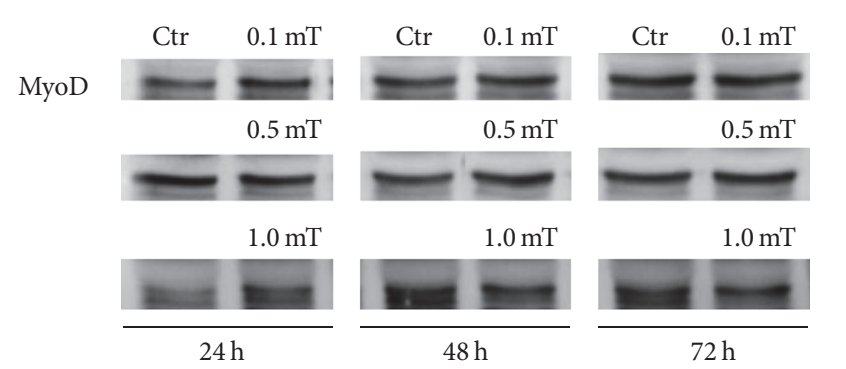

(a)

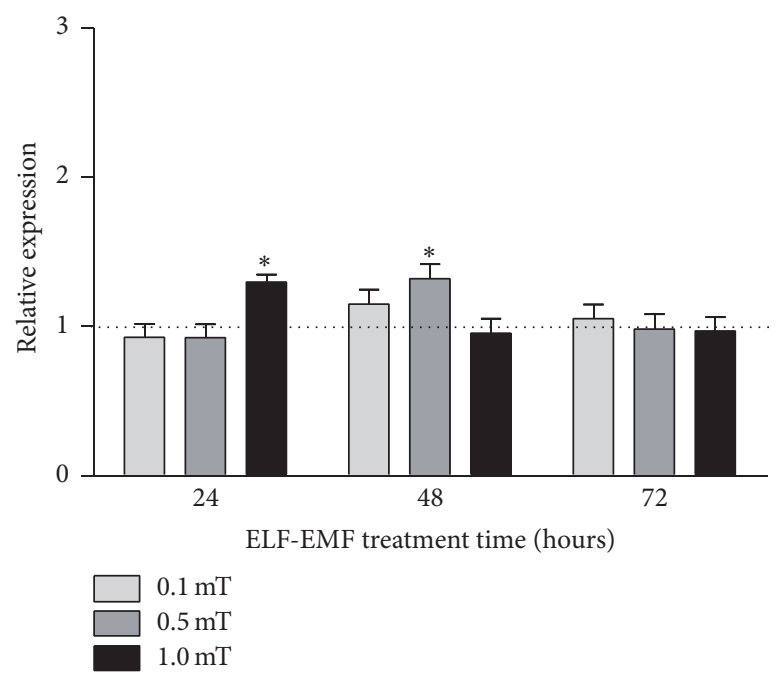

(b)

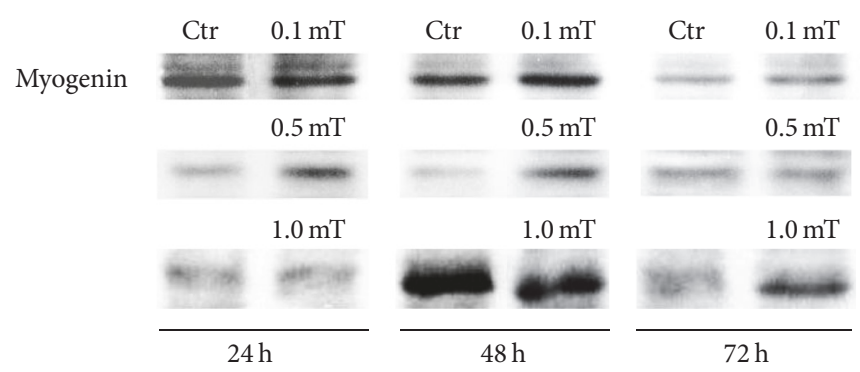

(c)

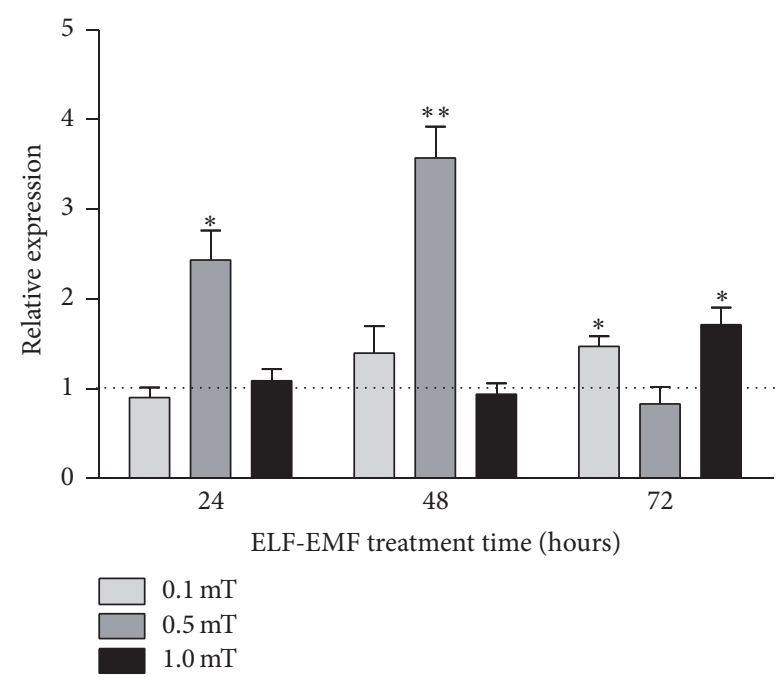

(d)

FIGURE 3: Expression levels of MyoD and myogenin in differentiating C2C12 myoblasts during ELF-EMF treatments. ((a) and (c)) Representative immunoblot of MyoD and myogenin expression levels, respectively. ((b) and (d)) Densitometry analysis of MyoD and myogenin expression levels, respectively, plotted as relative expression calculated by the ratio between $\mathrm{OD} \times \mathrm{mm}^{2}$ of $\mathrm{MyoD}$ or myogenin band found in ELF-EMF-treated cells $(0.1 \mathrm{mT}, 0.5 \mathrm{mT}$, or $1.0 \mathrm{mT})$ and $\mathrm{OD} \times \mathrm{mm}^{2}$ of the respective band in the corresponding control (Ctr). Data are means \pm SEM from three independent experiments. ${ }^{*} p<0.05$ and ${ }^{* *} p<0.01$ versus corresponding Ctr.

and biomedical researchers $[33,34]$. On the other hand, there is evidence of beneficial effects of magnetic fields and EMFs in the treatment of various injuries and diseases [9]. Indeed, clinical benefits for EMFs have been claimed for 20 centuries, and even if there has been perplexity and medical skepticism concerning their use, the application of EMFs with a therapeutic aim is probably one of the most ancient treatments used by Man [35].

Today, both static and time-varying magnetic fields are applied therapeutically with success in the treatment of musculoskeletal injuries or dysfunction [36]. There are also a large number of experimental and clinical studies that have demonstrated that various exogenous EMFs at surprisingly low levels can have effects on a variety of biological systems and processes, most of which are of critical importance for diagnostics and therapy. Along with the evidence from in vitro cellular models, clinical and preclinical studies have suggested that magnetic field and EMF stimulation can accelerate healing processes and influence nerve repair and regeneration [7].

Skeletal muscle also represents a potential target for the biological effect(s) of ELF-EMFs, and this property might be of great importance, due in particular to their diffuse medical applications in physical and rehabilitation medicine. To better clarify this point, the objective of the present study was to investigate the effects of ELF-EMFs on the myogenic process. To achieve this, the $\mathrm{C} 2 \mathrm{C} 12$ myoblastic cell line was chosen as a suitable in vitro model of skeletal muscle differentiation. The use of a well-characterized experimental model, the strict control of the experimental procedures, and the optimization of the protocol for this $\mathrm{C} 2 \mathrm{C} 12$ myoblast exposure to ELFEMFs have provided us with improved understanding of the cellular mechanism responsible for ELF-EMF-induced cell modifications, which have remained largely unknown to date.

The data presented here demonstrate that under these experimental conditions ELF-EMFs at $50 \mathrm{~Hz}$ and with electromagnetic field strengths from 0.1 to $1.0 \mathrm{mT}$ do not affect either $\mathrm{C} 2 \mathrm{C} 12$ myoblast viability or proliferation rate. This thus initially demonstrates that there are no direct toxic effects of ELF-EMFs on this cell model. Even if this finding is in apparent contrast with what has been observed with other cell models, it is important to bear in mind that, in the many studies that have reported that ELF-EMFs can act on cell proliferation and cell-cycle progression, these effects have depended 

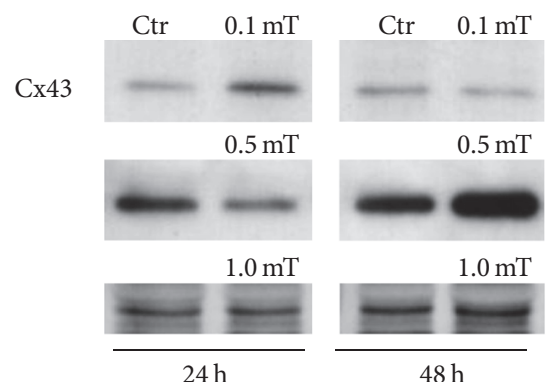

$48 \mathrm{~h}$

(a)

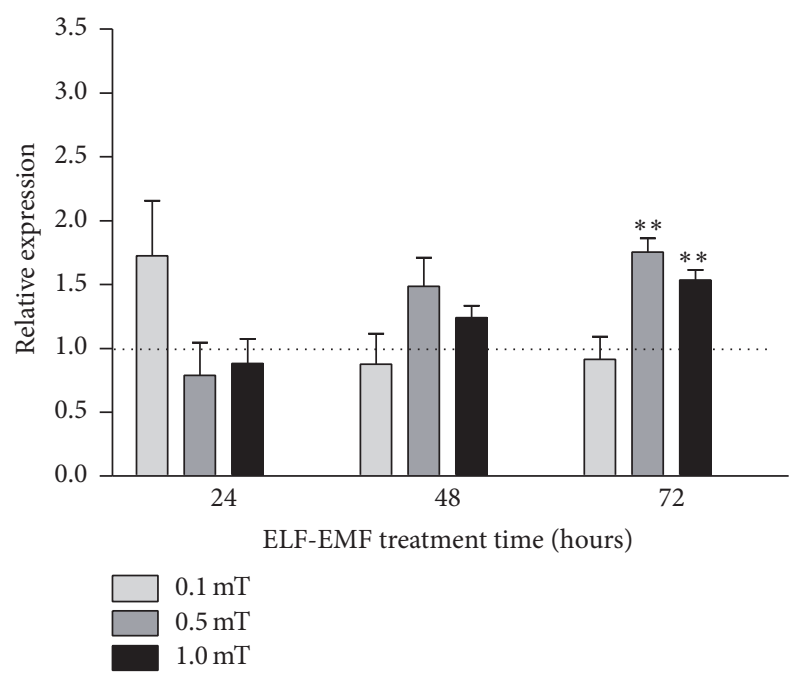

(b)

FIgURE 4: Expression levels of cx43 in differentiating C2C12 myoblasts during ELF-EMF treatments. (a) Representative immunoblot of cx43 expression levels, from three independent experiments. (b) Densitometry analyses plotted as relative expression calculated by the ratio between OD $\times \mathrm{mm}^{2}$ of the $\mathrm{cx} 43$ band found in ELF-EMFtreated cells $(0.1 \mathrm{mT}, 0.5 \mathrm{mT}$, and $1.0 \mathrm{mT})$, and $\mathrm{OD} \times \mathrm{mm}^{2}$ of the respective band in the corresponding control (Ctr). Data are means \pm SEM from three independent experiments ${ }^{* *} p<0.01$ versus corresponding Ctr.

on the cell phenotype, as well as on the intensity, frequency, and wave form of ELF-EMFs applied, and length of the exposure [37-42].

In recent decades, a large amount of evidence has demonstrated prodifferentiating effects of ELF-EMFs on different cell phenotypes, and interesting data have also been obtained using adult stem cells, with the suggestion that ELF-EMFs represent an efficacious therapeutic approach [28, 39, 43-45]. On the other hand, the mechanism(s) of action of ELF-EMFs on physiological processes and their intracellular molecular targets are far from being defined. In the cell model in the present study, the presence of ELF-EMFs enhanced the myogenic process. This process progresses through a highly ordered sequence of events, including morphological changes (i.e., cell alignment, fusion) and specific time-courses of gene expression patterns [46]. In addition, published data have strongly supported a key role for GJIC in the first phase of myogenic differentiation, when myoblasts that are committed
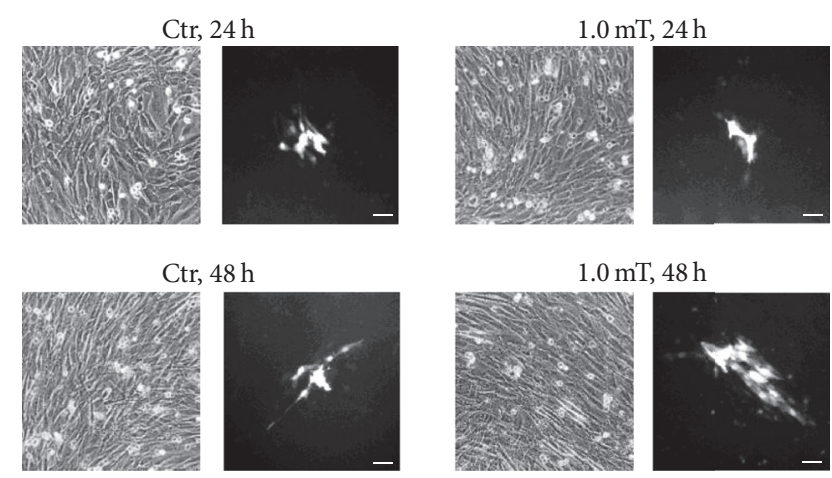

(a)

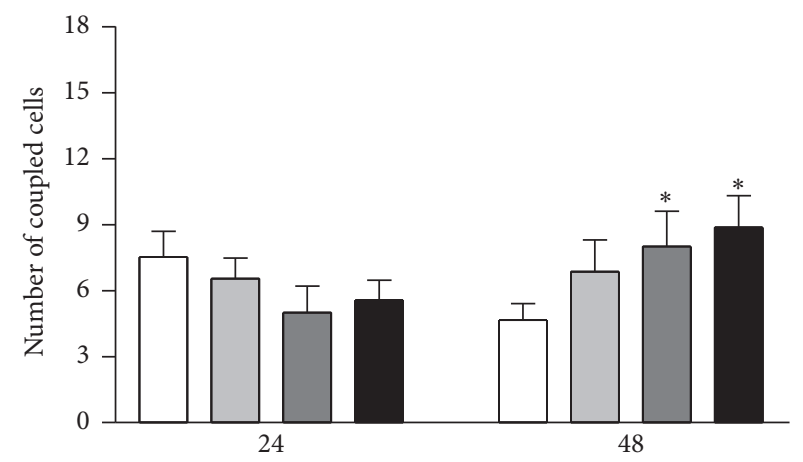

(h)


(b)

FIGURE 5: Functional coupling in differentiating C2C12 myoblasts during ELF-EMF treatments. (a) Representative phase contrast and corresponding fluorescence images of the $\mathrm{C} 2 \mathrm{C} 12$ myoblasts in the absence (Ctr) and presence of ELF-EMF treatment with $1.0 \mathrm{mT}$, from the beginning of the differentiation process ( $T 0$, time 0$)$ and after $24 \mathrm{~h}$ and $48 \mathrm{~h}$ in differentiation medium. Scale bars, $50 \mu \mathrm{m}$. (b) Quantification of dye-coupled cells under the conditions described in (a). Data are means \pm SEM from two independent experiments, each performed with at least 10 independent microinjections/plate. ${ }^{*} p<0.05$ versus corresponding Ctr.

to differentiate then adhere and fuse, to form gap junctions. These gap junctions then allow the intercellular diffusion of critical signals, such as $\mathrm{Ca}^{2+}$, inositol 1,4,5-trisphosphate, and adenosine $5^{\prime}$-triphosphate [24]. Indeed, when blockers of the permeability of these junction channels are used, or in the presence of inducible deletion of $\mathrm{cx} 43$, downregulation of myogenic factors is seen [21-23, 47]. Furthermore, transfection of rhabdomyosarcoma cells with cx43 cDNA was shown to induce cell differentiation [48]. In particular, in myoblasts, $\mathrm{cx} 43$ expression is predominant, and it is a prerequisite for their fusion [32]. Indeed, cx43 gap-junction channels have been shown to provide the intercellular signaling pathways that are required for normal timing of skeletal muscle ontogeny and regeneration [20]. In our cell model, ELF-EMFs at $0.1 \mathrm{mT}$ only transiently affected $\mathrm{cx} 43$ expression but at $0.5 \mathrm{mT}$ and $1.0 \mathrm{mT}$ modified GJIC activity through increases in both 
the expression of the cx43 protein and cell coupling in cultures committed to differentiate. These data are complementary to our previous report regarding the evidence that ELFEMFs can affect the $\mathrm{Ca}^{2+}$ handling and redox status of $\mathrm{C} 2 \mathrm{C} 12$ myoblasts [10]. These processes are responsible for the phenotypic cell reactions to environmental stimuli, and they might provide the means through which ELF-EMFs can accelerate the myogenesis process through increased extent of the GJIC, as the regulation of $\mathrm{Ca}^{2+}$ fluxes by gap junctions and their response to oxidative stress have been well demonstrated [49].

\section{Conclusions}

In conclusion, although our data do not show clear dosedependence or threshold-induced effects, ELF-EMFs in the $\mathrm{mT}$ intensity range accelerated the myogenic process in these C2C12 myoblasts. In the same cell model, ELF-EMFs can affect the cellular redox state and intracellular $\mathrm{Ca}^{2+}$ dynamics [10] and, consequently, cell metabolic activity. All of these events can be proposed as key triggers involved in increases in specific myogenic markers (e.g., MyoD and myogenin) and the promotion of cell fusion, which is made more efficient by the increased GJIC activity, all of which are steps that can be enhanced by ELF-EMFs.

Taken together, all these evidences indicate for the first time the mechanism of action through which ELF-EMFs can promote skeletal muscle differentiation. This thus provides a scientific basis to what is considered an efficacious therapeutic means to resolve, at least in part, some conditions of muscle dysfunction.

\section{Disclosure}

Giovanna Mazzoleni and Maria A. Mariggiò are co-senior authors.

\section{Conflicts of Interest}

The authors report no conflicts of interest.

\section{Authors' Contributions}

Caterina Morabito, Nathalie Steimberg, Francesca Rovetta, Giovanna Mazzoleni, and Maria A. Mariggiò conceived and designed the experiments and contributed to the writing of the manuscript. Caterina Morabito, Nathalie Steimberg, Francesca Rovetta, Simone Guarnieri, Jennifer Boniotti, and Giovanna Mazzoleni performed the experiments. Caterina Morabito, Nathalie Steimberg, Francesca Rovetta, Simone Guarnieri, and Jennifer Boniotti analyzed the data. Caterina Morabito and Nathalie Steimberg contributed equally to this study.

\section{Acknowledgments}

This study was supported by "G. d'Annunzio" University research funds and in part by ASI (Grant no. 2014-018-R.O to Maria A. Mariggiò), by MATT (Grants 5376/2002/SIAR and DSA/2004/0026772), and by local research funds of the University of Brescia to Giovanna Mazzoleni.

\section{References}

[1] A. Ahlbom and M. Feychting, "Electromagnetic radiation," British Medical Bulletin, vol. 68, pp. 157-165, 2003.

[2] M. S. Markov, "Effects of electromagnetic fields on biological systems," Electromagnetic Biology and Medicine, vol. 32, no. 2, pp. 121-122, 2013.

[3] L. Kheifets, A. Ahlbom, C. M. Crespi et al., "Pooled analysis of recent studies on magnetic fields and childhood leukaemia," British Journal of Cancer, vol. 103, no. 7, pp. 1128-1135, 2010.

[4] D. A. Savitz, H. Wachtel, F. A. Barnes, E. M. John, and J. G. Tvrdik, "Case-control study of childhood cancer and exposure to 60-Hz magnetic fields," The American Journal of Epidemiology, vol. 128, no. 1, pp. 21-38, 1988.

[5] J. Schüz and A. Ahlbom, "Exposure to electromagnetic fields and the risk of childhood leukaemia: A review," Radiation Protection Dosimetry, vol. 132, no. 2, pp. 202-211, 2008.

[6] Vijayalaxmi and G. Obe, "Controversial cytogenetic observations in mammalian somatic cells exposed to extremely low frequency electromagnetic radiation: A review and future research recommendations," Bioelectromagnetics, vol. 26, no. 5, pp. 412-430, 2005.

[7] M. S. Markov, "Expanding use of pulsed electromagnetic field therapies," Electromagnetic Biology \& Medicine, vol. 26, no. 3, pp. 257-274, 2007.

[8] M. S. Markov, "Magnetic field therapy: a review," Electromagn Biol Med, vol. 26, no. 1, pp. 1-23, 2007.

[9] M. S. Markov and A. P. Colbert, "Magnetic and electromagnetic field therapy," Journal of Back and Musculoskeletal Rehabilitation, vol. 15, no. 1, pp. 17-29, 2000.

[10] C. Morabito, F. Rovetta, M. Bizzarri, G. Mazzoleni, G. Fanò, and M. A. Mariggiò, "Modulation of redox status and calcium handling by extremely low frequency electromagnetic fields in C2C12 muscle cells: A real-time, single-cell approach," Free Radical Biology and Medicine, vol. 48, no. 4, pp. 579-589, 2010.

[11] D. Yaffe and O. Saxel, "Serial passaging and differentiation of myogenic cells isolated from dystrophic mouse muscle," Nature, vol. 270, no. 5639, pp. 725-727, 1977.

[12] V. E. Jahnke, O. Sabido, and D. Freyssenet, "Control of mitochondrial biogenesis, ROS level, and cytosolic Ca 2+ concentration during the cell cycle and the onset of differentiation in L6E9 myoblasts," American Journal of Physiology - Cell Physiology, vol. 296, no. 5, pp. C1185-C1194, 2009.

[13] M. H. Parker, P. Seale, and M. A. Rudnicki, "Looking back to the embryo: defining transcriptional networks in adult myogenesis," Nature Reviews Genetics, vol. 4, no. 7, pp. 497-507, 2003.

[14] R. L. Perry and M. A. Rudnick, "Molecular mechanisms regulating myogenic determination and differentiation," Frontiers in Bioscience, vol. 5, pp. D750-D767, 2000.

[15] K. Yun and B. Wold, "Skeletal muscle determination and differentiation: Story of a core regulatory network and its context," Current Opinion in Cell Biology, vol. 8, no. 6, pp. 877-889, 1996.

[16] J. C. J. Chen and D. J. Goldhamer, "Skeletal muscle stem cells," Reproductive Biology and Endocrinology, vol. 1, article no. 101, 2003.

[17] I. Gonzalez, G. Tripathi, E. J. Carter et al., "Akt2, a Novel Functional Link between p38 Mitogen-Activated Protein Kinase 
and Phosphatidylinositol 3-Kinase Pathways in Myogenesis," Molecular and Cellular Biology, vol. 24, no. 9, pp. 3607-3622, 2004.

[18] T. J. Hawke and D. J. Garry, "Myogenic satellite cells: physiology to molecular biology," Journal of Applied Physiology, vol. 91, no. 2, pp. 534-551, 2001.

[19] M. I. Kontaridis, X. Liu, L. Zhang, and A. M. Bennett, "Role of SHP-2 in fibroblast growth factor receptor-mediated suppression of myogenesis in C2C12 myoblasts," Molecular and Cellular Biology, vol. 22, no. 11, pp. 3875-3891, 2002.

[20] R. Araya, D. Eckardt, S. Maxeiner et al., "Expression of connexins during differentiation and regeneration of skeletal muscle: Functional relevance of connexin43," Journal of Cell Science, vol. 118, no. 1, pp. 27-37, 2005.

[21] R. Araya, D. Eckardt, M. A. Riquelme, K. Willecke, and J. C. Saez, "Presence and importance of connexin43 during myogenesis," Cell Communication \& Adhesion, vol. 10, no. 4-6, pp. 451456, 2003.

[22] B. Constantin, L. Cronier, and G. Raymond, "Transient involvement of gap junctional communication before fusion of newborn rat myoblasts," Comptes Rendus de l'Academie des Sciences - Serie III, vol. 320, no. 1, pp. 35-40, 1997.

[23] R. M. Mège, D. Goudou, C. Giaume, M. Nicolet, and F. Rieger, "Is intercellular communication via Gap junctions required for myoblast fusion?" Cell Communication and Adhesion, vol. 2, no. 4, pp. 329-343, 1994.

[24] B. Constantin and L. Cronier, "Involvement of gap junctional communication in myogenesis," International Review of Cytology, vol. 196, pp. 1-65, 2000.

[25] A. Gorbe, D. L. Becker, L. Dux et al., "Transient upregulation of connexin43 gap junctions and synchronized cell cycle control precede myoblast fusion in regenerating skeletal muscle in vivo," Histochemistry and Cell Biology, vol. 123, no. 6, pp. 573-583, 2005.

[26] J. von Maltzahn, V. Wulf, and K. Willecke, "Spatiotemporal expression of connexin 39 and -43 during myoblast differentiation in cultured cells and in the mouse embryo," Cell Communication and Adhesion, vol. 13, no. 1-2, pp. 55-60, 2006.

[27] M. Farina, M. Farina, M. A. Mariggiò et al., "ELF-EMFs induced effects on cell lines: Controlling ELF generation in laboratory," Progress In Electromagnetics Research B, no. 24, pp. 131-153, 2010.

[28] C. Morabito, S. Guarnieri, G. Fanò, and M. A. Mariggiò, "Effects of acute and chronic low frequency electromagnetic field exposure on PC12 cells during neuronal differentiation," Cellular Physiology and Biochemistry, vol. 26, no. 6, pp. 947-958, 2010.

[29] S. Guarnieri, C. Morabito, S. Belia et al., "New insights into the relationship between mIGF-1-induced hypertrophy and $\mathrm{Ca} 2+$ handling in differentiated satellite cells," PLoS ONE, vol. 9, no. 9, p. e107753, 2014.

[30] G. Mazzoleni, P. Telo, A. Camplani, S. Tanganelli, S. Monarca, and G. Ragnotti, "Influence of the herbicide Linuron on growth rate and gap-junctional intercellular communication of cultured endothelial cells," Journal of Environmental Pathology, Toxicology and Oncology, vol. 13, no. 1, pp. 1-10, 1994.

[31] D. Nosi, R. Mercatelli, F. Chellini et al., "A molecular imaging analysis of $\mathrm{Cx} 43$ association with Cdo during skeletal myoblast differentiation," Journal of Biophotonics, vol. 6, no. 8, pp. 612621, 2013.

[32] R. Squecco, C. Sassoli, F. Nuti et al., "Sphingosine 1-phosphate induces myoblast differentiation through $\mathrm{Cx} 43$ protein expression: A role for a gap junction-dependent and -independent function," Molecular Biology of the Cell, vol. 17, no. 11, pp. 48964910, 2006.

[33] R. M. Macklis, "Magnetic healing, quackery, and the debate about the health effects of electromagnetic fields," Annals of Internal Medicine, vol. 118, no. 5, pp. 376-383, 1993.

[34] M. Maslanyj, T. Lightfoot, J. Schüz, Z. Sienkiewicz, and A. McKinlay, "A precautionary public health protection strategy for the possible risk of childhood leukaemia from exposure to power frequency magnetic fields," BMC Public Health, vol. 10, article no. 673, 2010.

[35] J. R. Basford, "A historical perspective of the popular use of electric and magnetic therapy," Archives of Physical Medicine and Rehabilitation, vol. 82, no. 9, pp. 1261-1269, 2001.

[36] C. T. Brighton, B. Strafford, S. B. Gross, D. F. Leatherwood, J. L. Williams, and Pollack., "SR: The proliferative and synthetic response of isolated calvarial bone cells of rats to cyclic biaxial mechanical strain," The Journal of Bone and Joint Surgery. American, vol. 73, no. 3, pp. 320-331, 1991.

[37] I. Y. Belyaev and E. D. Alipov, "Frequency-dependent effects of ELF magnetic field on chromatin conformation in Escherichia coli cells and human lymphocytes," Biochimica et Biophysica Acta-General Subjects, vol. 1526, no. 3, pp. 269-276, 2001.

[38] S. Falone, S. Jr Santini, S. di Loreto et al., "Improved mitochondrial and methylglyoxal-related metabolisms support hyperproliferation induced by $50 \mathrm{~Hz}$ magnetic field in neuroblastoma cells," Journal of Cellular Physiology, vol. 231, no. 9, pp. 20142025, 2016.

[39] A. Maziarz, B. Kocan, M. Bester et al., "How electromagnetic fields can influence adult stem cells: Positive and negative impacts," Stem Cell Research and Therapy, vol. 7, no. 1, article no. $54,2016$.

[40] M. Simko, "Induction of cell activation processes by low frequency electromagnetic fields," Scientific World Journal, vol. 4, 2, pp. 4-22, 2004.

[41] A. R. Sul, S.-N. Park, and H. Suh, "Effects of sinusoidal eletromagnetic field on stucture and function of different kinds of cell lines," Yonsei Medical Journal, vol. 47, no. 6, pp. 852-861, 2006.

[42] M. Sulpizio, S. Falone, F. Amicarelli et al., "Molecular basis underlying the biological effects elicited by extremely low-frequency magnetic field (ELF-MF) on neuroblastoma cells," Journal of Cellular Biochemistry, vol. 112, no. 12, pp. 3797-3806, 2011.

[43] R. H. W. Funk and T. K. Monsees, "Effects of electromagnetic fields on cells: Physiological and therapeutical approaches and molecular mechanisms of interaction. A review," Cells Tissues Organs, vol. 182, no. 2, pp. 59-78, 2006.

[44] A. Lisi, A. Foletti, M. Ledda et al., "Extremely low frequency 7 $\mathrm{Hz} 100 \mu$ t electromagnetic radiation promotes differentiation in the human epithelial cell line HaCaT,' Electromagnetic Biology and Medicine, vol. 25, no. 4, pp. 269-280, 2006.

[45] T. Saliev, Z. Mustapova, G. Kulsharova, D. Bulanin, and S. Mikhalovsky, "Therapeutic potential of electromagnetic fields for tissue engineering and wound healing," Cell Proliferation, vol. 47, no. 6, pp. 485-493, 2014.

[46] V. Andrés and K. Walsh, "Myogenin expression, cell cycle withdrawal, and phenotypic differentiation are temporally separable events that precede cell fusion upon myogenesis," Journal of Cell Biology, vol. 132, no. 4, pp. 657-666, 1996.

[47] A. Proulx, P. A. Merrifield, and C. C. G. Naus, "Blocking gap junctional intercellular communication in myoblasts inhibits myogenin and MRF4 expression," Developmental Genetics, vol. 20, no. 2, pp. 133-144, 1997. 
[48] A. A. Proulx, Z. X. Lin, and C. C. Naus, "Transfection of rhabdomyosarcoma cells with connexin 43 induces myogenic differentiation," Cell Growth and Differentiation, vol. 8, no. 5, pp. 533540, 1997.

[49] J. C. Sáez, K. A. Schalper, M. A. Retamal, J. A. Orellana, K. F. Shoji, and M. V. L. Bennett, "Cell membrane permeabilization via connexin hemichannels in living and dying cells," Experimental Cell Research, vol. 316, no. 15, pp. 2377-2389, 2010. 




The Scientific World Journal
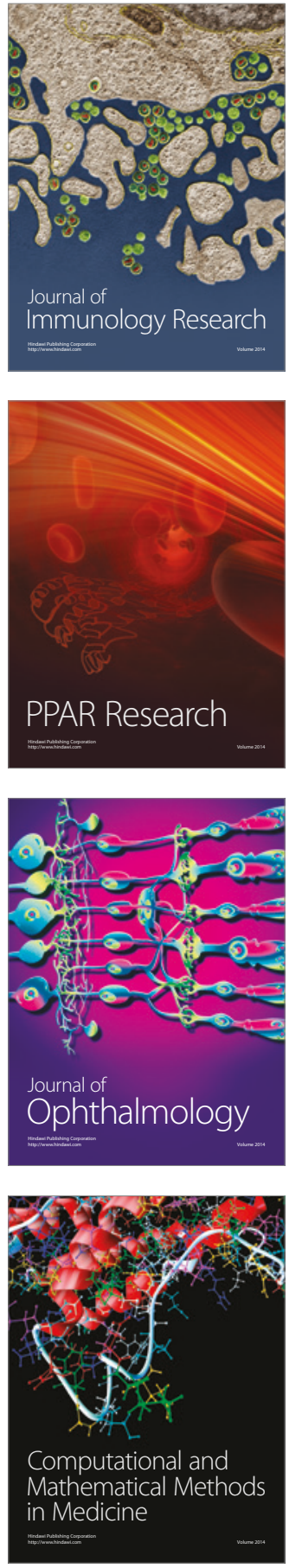

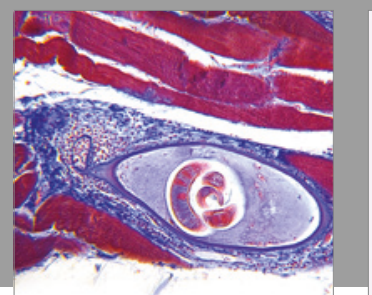

Gastroenterology Research and Practice
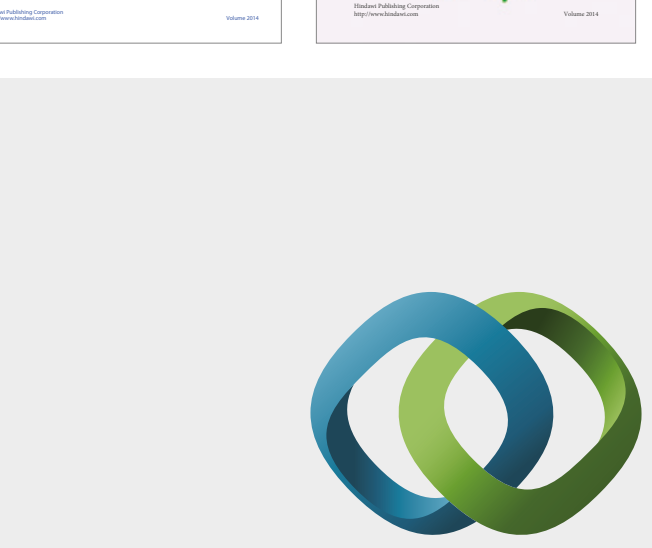

\section{Hindawi}

Submit your manuscripts at

https://www.hindawi.com
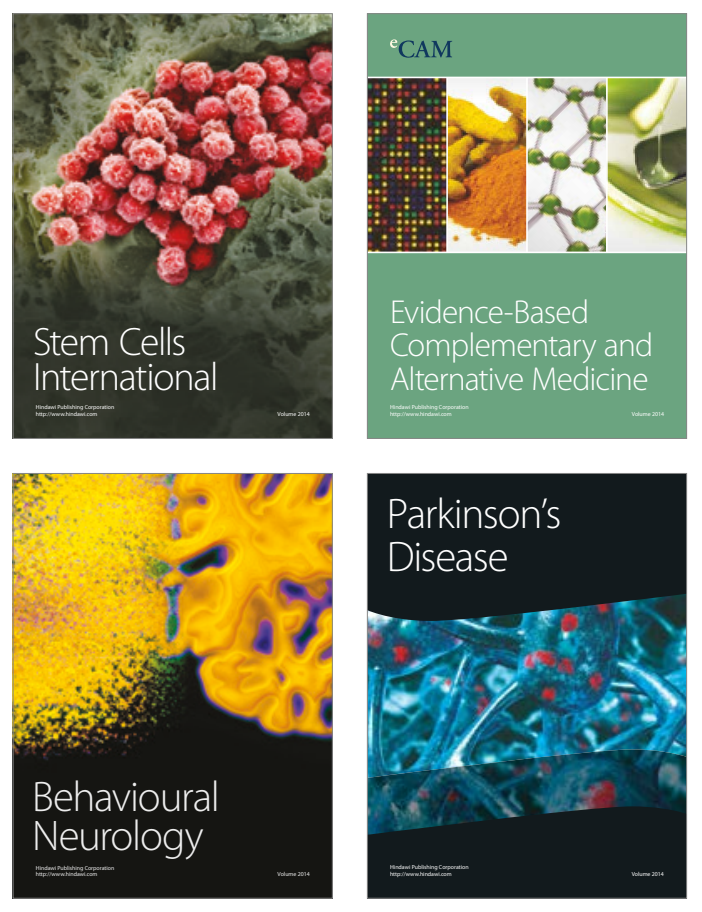
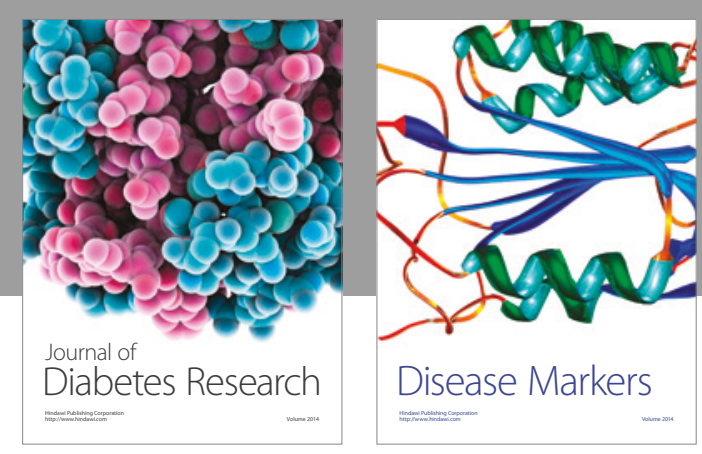

Disease Markers
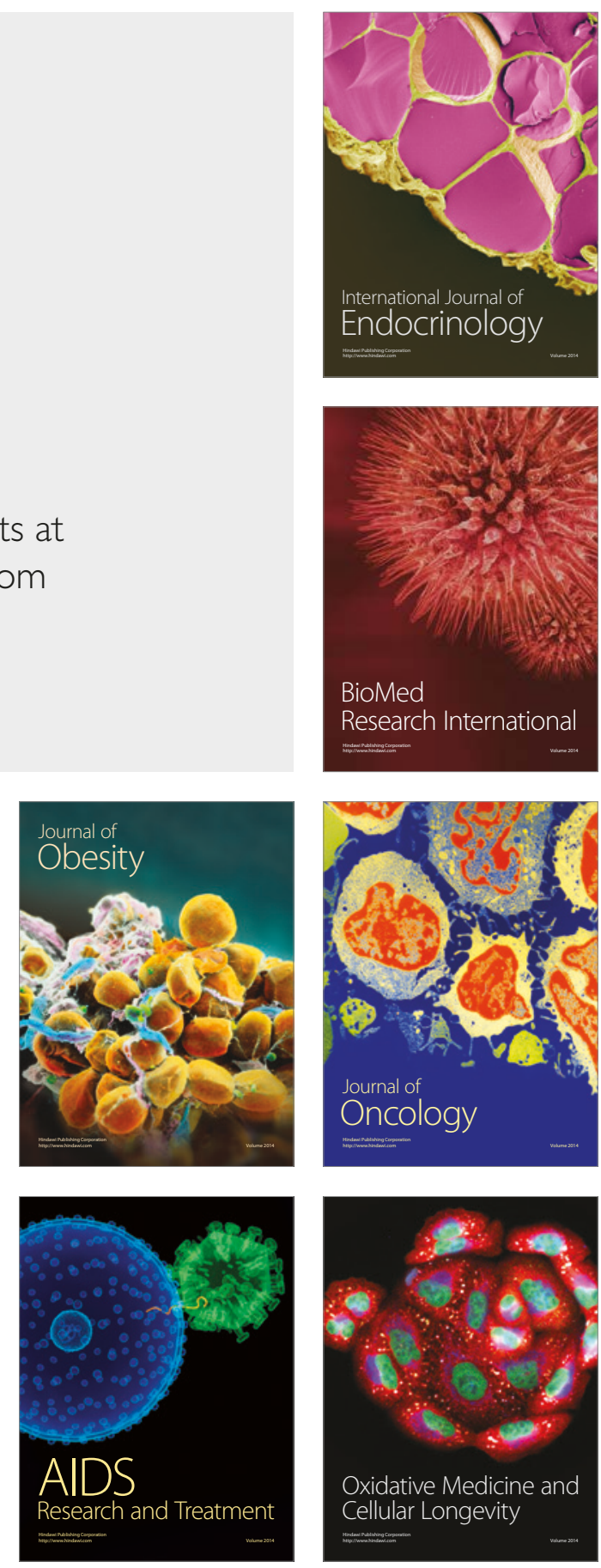